\title{
Conference Paper Modeling Cardiac Congenital Diseases: From Mathematic Tools to Human Induced Pluripotent Stem Cells
}

\author{
Laura Iop \\ Department of Cardiac, Thoracic and Vascular Sciences, University of Padua Medical School, Via Giustiniani 2, 35128 Padua, Italy \\ Correspondence should be addressed to Laura Iop; laura.iop@unipd.it
}

Received 22 November 2013; Accepted 9 March 2014; Published 12 May 2014

Academic Editors: V. La Carrubba, A. Lepedda, and J. C. Rodriguez-Cabello

This Conference Paper is based on a presentation given by Laura Iop at "LIAC Meeting on Vascular Research 2013 " held from 18 September 2013 to 21 September 2013 in Alghero, Italy.

Copyright (C) 2014 Laura Iop. This is an open access article distributed under the Creative Commons Attribution License, which permits unrestricted use, distribution, and reproduction in any medium, provided the original work is properly cited.

Cardiac congenital diseases are rare inherited disorders characterized by anatomical malformations and/or by electrophysiological abnormalities, both affecting the whole heart function. In order to clarify the underlying pathophysiological mechanisms, experimental modeling has been proposed through in silico, in vitro, and/or in vivo simulations. Bioinformatics, transgenesis, heterologous expression systems, mammalian models, and, recently, pluripotent stem cells have been advanced to effectively recapitulate several human congenital diseases (such as Brugada syndrome, CPVT, LQTs, and ARVC) and, potentially, provide new insights into their pathomechanisms for novel therapeutic perspectives.

\section{Introduction}

Cardiac congenital diseases are malformations of the heart, with lethal consequences in the fetal life or able to compromise the cardiovascular function often soon after birth. From simpler forms, as the atrial septal defects, to more complex abnormalities, as the complete atrioventricular canal and the Tetralogy of Fallot, such class of structural heart diseases has incidence per 1000 live births varying from an average 6.9 in Europe to 9.3 in Asia or 8 in the United States. The more severe symptoms can manifest in the first year of life: frequently, they are recognized only after infant death or when promptly diagnosed, require urgent surgical correction in approximately $25 \%$ cases. They might also be asymptomatic until the adult age in the so-called GUCH population (grown-up congenital heart) [1].

In other cases, congenital cardiac diseases are not related to morpho-anatomical defects of the heart but caused by mutations in important proteins related to its global function. In macroscopically normal hearts, ion channels, transporters, and other accessory proteins can be interested by molecular anomalies affecting their cardinal properties, thus resulting in a deregulation of the normal electromechanical coupling. Encoded by almost 350 genes, the large family of ion channels comprises proteins with a unique domain or multiple subunits, arranged in different topologies at the cell membrane. Owing to precise selective and gating features, they allow the passive flow of specific ions through their pores across the membrane interface and differ from ion pumps or sym/antiporters since they do not require energy to regulate the electrochemical movement. Many accessory proteins tune the biophysical activities of these molecular switches either as their subdomains or as specialized chaperone or anchoring units [2]. In the cardiomyocyte electromechanical machine, such proteins concur during the action potential (AP) to the final release of calcium from the sarcoplasmic reticulum into the cytosol, where it reaches the myofilaments and activates the contraction in the systole. During the AP last phase in diastole, they participate in the reuptake of calcium ions and their sequestration in the sarcoplasmic reticulum for the repolarization of the cardiac myocyte [3].

If any of the proteins involved in this machinery is malfunctioning, the equilibrium of ionic currents or the contraction of the myofibrils result altered and arrhythmic events are generated submitting the subject to ventricular fibrillation, palpitations, tachyarrhythmia, syncope, and, in the worst case, sudden death. 
In the identification of the genetic substrate of such inherited arrhythmias, the molecular culprits of these pathological phenotypes have to be searched mostly in mutations of genes codifying for ion channels. Several syndromes, for example, Brugada and multiple subtypes of long QTs, are related to congenital diseases of a specific ion channel, the so-called channelopathy. In other cases, as in the arrhythmogenic right ventricular cardiomyopathy/dysplasia, the cardiac muscle disease is provoked by a single mutation in intercellular junction proteins.

Experimental modeling has been proposed to fully elucidate the pathophysiological mechanisms underlying these cardiac monogenic diseases and eventually to design novel and more appropriate therapies.

Bioinformatics, transgenesis, heterologous expression systems, mammalian models, and recently, pluripotent stem cells have been used as tools to experimentally reproduce the human disorder and provide new insights into its pathological mechanism and possible treatment.

\section{Experimental Approaches to Modeling}

2.1. Mathematical and Bioinformatic Simulations. Mathematic modeling has been applied to the field of cardiac electrophysiology since the 50es of the previous century to introduce a more comprehensive picture on the functional behavior or interactions of the different proteins involved in the cell electrical activities.

Initially, such bioinformatic simulations aimed at depicting the functioning of a single transmembrane ion channel in respect to its gating and conductance properties, following approximations by Hodgkin and Huxley in regard to axonal cells [4]. The adjustment of this model with experimental data about state dependent transitions allowed the formulation of Markov paradigm, which could more realistically reproduce ion channel kinetics variations related to a mutated gene [5].

A further degree of complexity has been added with the simulated intracellular ion dynamics, reproducing calcium handling in all its components (release from the sarcoplasmic reticulum, cytosol diffusion, and further reuptake) and interested coplayers.

The modeling of a cardiac cell needs indeed to take into account the large heterogeneity of tissue specialization and species origin (for further details on this aspect see "In vitro and in vivo animal models" section dedicated to this topic). So far attempts to simulate a cardiac electric unit are manifold, as summarized by a recent comprehensive review [6]. FitzHugh and Noble models were the first to be proposed in 1960-1962. Both models elaborated the Hodgkin/Huxley simulation, respectively, by predicting the existence of a persistent sodium inward current (sodium window current) in the generation of the plateau phase and by introducing the potassium inward current, lately identified as rectifier $I_{\mathrm{K} 1}$, and its key-role in the potassium gradient-dependent action potential velocity. The pitfalls of these first models resided in the too high ion conductance of the first and in the evaluation of a unique modality of voltage gating in $I_{\mathrm{Na}}$ current for the second [7]. Nearly fifteen years later, McAllister, Noble, and Tsien implemented the first models with other information obtained experimentally for the electrophysiological description of the Purkinje cell. Such model accounted 10 variables, namely the membrane potential and 9 gates with activation, inactivation or plateau behavior for sodium, potassium or chloride currents [8]. Beeler and Reuter formulated the first model of ventricular AP by introducing 4 currents, 2 inward, i.e., $I_{\mathrm{Na}}$, with its fast and slow inactivation parameters and calcium $I_{S}$ and 2 outward, i.e., rectifier $I_{\mathrm{K} 1}$ and voltage- and time-dependent $I_{\mathrm{X} 1}$ [9]. In 1982, DiFrancesco et al. discovered the typical pacemaker or funny current $I_{f}$ : this was integrated in a novel simulation with ion channels and exchangers, among which the $\mathrm{Na}-\mathrm{K}$ pump is directly implicated in the $I_{f}$ generation $[10,11]$. The absence of calcium buffering in this model had been overcome introducing a voltage-dependent calcium release with fast transient. Since 1991, Luo and Rudy improved their early passive model to a late dynamic simulation. It was hence possible to foresee the current modifications on the basis of the dynamic concentration shifts of their ions and to represent a high number of single ion channels, heterogeneous in tissue specialization, following Markov modeling $[12,13]$. As the previous model, Ten Tusscher's one had rendered feasible more recently to illustrate human cells derived from different heart tissues by including 12 currents and 9 variables, in particular calcium handling [14].

The progressive implementation of predictive models with experimental evidences has allowed the generation of sophisticated integrative replicas, able also to mimic different protein states, as well as protein-protein interactions in several cell and tissue types.

In the attempt to provide organ-level modeling, multiscale mechanical and electrophysiological computations have been integrated taking into consideration myofilament behavior [15], calcium handling, and the mechanical effects induced by calcium link to troponin in terms of sarcomere length and whole organ deformation [16, 17]. Such complex electromechanical models are particularly useful in the prediction of the whole organ effects during arrhythmic conditions, caused by ion channel mutations, and also after applied therapies for their treatment [18].

While showing such essentiality, these bioinformatics tools may fail to really recapitulate the natural electrophysiological activities of heart proteins, cells, and tissues, due to their intrinsic predictive nature and even if logically formulated, they can incur in denial following experimental observations.

2.2. Heterologous Expression Systems. In the search to analyze native protein functionality in vitro, biological systems have been engineered for heterologous expression. Yeasts, especially Pichia pastoris and Saccharomyces cerevisiae, have been widely used to express human proteins with the advantages of rapid growth, well-known genetics, and cost-effectiveness. In particular, the yeast two-hybrid approach has allowed through the genetic engineering of yeast cells to evaluate the interactions between a couple of proteins, particularly relevant in the case of a mutation [19]. Xenopus laevis oocytes have also been engineered on this purpose, especially for the possibility to approximate this system to an in vivo model 
$[20,21]$. However, the cell biology of this animal features some traits of inadequacy in mimicking mammalian cells because of unshared mechanisms of protein maturation and working temperature [22].

For superior proximity to the human system, some mammalian cell lines, such as COS-7 and HEK293, respectively, derived from monkey and human kidney, have been employed as expression system of cardiac myocyte proteins involved in the electromechanical coupling [23, 24]. As noncardiac cells, HEK293- or COS7-based heterologous expression systems can be useful in the analyses of single ion channels in their native or mutated forms, but do not represent a precise electrophysiological phenocopy of the whole cardiomyocyte.

2.3. In Vitro and In Vivo Animal Models. To overcome the unfeasible integration of all ionic currents related to nonmammalian and/or noncardiac cells, atrial, ventricular, and Purkinje cells have been isolated from the hearts of mice and rabbits especially $[25,26]$. In addition, mutations can be stably introduced by genetic engineering through welldeveloped manipulation techniques in mammalian cells.

Cardiac primary cell cultures are obtained through enzymatic dissociation of specific tissues or whole organ. Such procedure allows for the achievement of a pure myocyte population excluding other cell contaminants, but may induce damages in cell functionality, particularly at the surface level. Experiments, even long term, can be performed only after recovery in controlled culturing conditions and in presence of opportune petri dish precoating with extracellular matrix proteins, as laminin or fibronectin, to favor cell adhesion. These settings are relatively critical for the survival and phenotype maintenance of isolated cardiac myocytes. Adhering cardiomyocytes require a certain degree of adaptability and modulation of all their physiological properties to the novel, artificial microenvironment: this phenomenon is usually described as dedifferentiation, that is, the reversion to an embryonic-like phenotype characterized by spontaneous contractions and expression of beta myosin heavy chains nonmanifested in vivo [27].

Veterinary studies have revealed that many cardiac congenital diseases similar to human pathologies are also suffered by mammals. For instance, cats and boxer dogs can be observed for the study of arrhythmogenic right ventricular cardiomyopathy $[28,29]$.

Transgenesis has offered the invaluable opportunity to create novel in vivo disease models by inducing gain or loss of function of specific proteins. Knockout and knock-in strategies have been applied to introduce the specific mutated form of the interest gene in the genome of animal embryonic stem cells. The injection of the latter in the blastocyst of another animal generates a chimera. Once implanted into the uterus of a female animal, procreated male chimeric offspring crossed with a normal female animal gives rise to heterozygous mutant progeny. Mutants in homozygosis are generated after inbreeding.

Several transgenic animals have been generated to study the pathophysiology of inherited rhythm disturbances. Rabbits have been transgenically modified for the complete abolishment of $I_{\mathrm{Ks}}$ and $I_{\mathrm{Kr}}$ potassium currents [30]. Genetic manipulations in rodents generated mice with complete suppression of $I_{\mathrm{Na}}$ sodium currents, $I_{\mathrm{to}, f}$ and $I_{\mathrm{to}, s}$ transient outward potassium currents, or deletion of connexins and plakophilin 2 desmosomal protein [31-34].

In respect to the human heart electrophysiology, cells or organs of other mammals possess some degrees of discrepancy, as assessed in dog and guinea pig ventricular myocytes in a recent study by O'Hara and Rudy [35].

As natural carriers of arrhythmic disturbances, dogs have been selected as interesting models to study the pathophysiologic effects of such diseases [29].

While pigs are often used as experimental model for chemically induced ventricular fibrillation [36], they completely lack the expression of $I_{\text {to }} \mathrm{K}^{+}$currents [37].

One of the closest animal models to men is the rabbit, sharing very similar action potentials, even if the smaller size of the heart cannot fully reproduce the human global electromechanical behavior.

Generally, small kinetics variations are responsible for the different AP waveforms registered in interspecies comparisons [25].

Rodents display particular electrophysiological characteristics, which render them very dissimilar to humans. Actually, mice and rats have a shorter duration of the action potential with a distinctly rapid repolarization phase and a resting heart frequency able to reach nearly 700 beats per minute. Marked differences can regard the distribution and activities of potassium currents. Even if clinical signs of most human channelopathies are well reproduced in transgenic rodents, engineered mice for the expression of potassium channel mutations never developed the sustained arrhythmias observed in affected men [38].

\subsection{In Vitro Studies with Cardiac Cells Harvested from the} Heart of Affected Patients. Native ion channels from the human heart represent the best choice for a perfect match mutation-phenotype. Endomyocardial biopsy is a routine clinical procedure in heart allotransplantation surveillance, as well as for the diagnosis of inflammatory myocardial diseases and unexplained heart failure. With such procedure 4-5 heart samples are obtained using dedicated bioptomes [39]. Nevertheless, the procedure is particularly invasive and can seriously compromise an already precarious clinical condition characterized by malignant arrhythmias.

As for animal hearts, protocols for the isolation of primary cardiomyocytes have been optimized also starting from these modest human specimens [40]. The relatively low yield on primary cells achievable from such biopsies restricts the number of possible experimental studies to be performed in vitro. In addition, cell instability of adult cardiac myocytes represents another issue in the use of human heart-derived cells for in vitro research.

\subsection{Human Embryonic Stem Cells and Induced Pluripotent} Stem Cells. Embryonic stem cells (ESCs) are native cells with the ability of self-renewal and transdifferentiation into all three germ layers and therefore giving rise to all the 
cells of the developing body. Thomson first isolated ESCs from the inner mass of the human blastocyst in 1998, after the achievement of correspondent cells from several species embryos [41]. Moreover, ESCs are commonly used in the generation of transgenic animals after the introduction of a specific mutation in their genome by genetic engineering.

Nearly ten years later, Takahashi and Yamanaka reported the in vitro development of a novel cell line with ESC-like pluripotent stem biology [42]. Mouse adult differentiated fibroblasts, obtained from a skin biopsy, were forced to reactivate the embryonic developmental pathways by retrovirusmediated introduction of four transcription factor transgenes (c-myc, Klf4, Sox2, and Oct-4). Such manipulation was able to induce a pluripotent state in treated fibroblast cells, newly called mouse induced pluripotent stem cells (iPSCs). In 2007, Takahashi et al. generated human iPSC lines starting from the same human adult cell type [43]. Thanks to the recent advance in genetic engineering technology, iPSCs have been developed with more accessible cell types, as peripheral T-lymphocytes, along with nonintegrating reprogramming systems, such as Sendai virus or small molecules [44, 45].

Several protocols have been established to differentiate these pluripotent stem cells towards cardiac myocytes and other vascular cells [46-48], paving the way for their application in many fields of cardiovascular medicine. Human iPSCs are particularly strategic in respect to ESCs, since they can be generated also from patient cells. In addition, their adult derivation eschews ethical controversies related to the use of human embryos for research purposes (forbidden in Italy, as in other European countries). In addition, the obtainment of iPSCs from patient's cells allows analyzing the inherited pathology directly in the petri dish as further investigative tool in respect to clinical routine management.

It is thus possible to evaluate the distribution pattern of the mutated protein to evidence trafficking problems and assembly at the cell membranes, as well as to assess the behavior of the specific ion currents composing the action potentials.

Besides the study on the disease mechanisms, such approach renders feasible also the reliable screening of novel chemical compounds, as well as the identification of effective drug dosages for the development of more personalized treatments.

Fundamental to mention is indeed the promise of the application of iPSCs in the cardiovascular regenerative medicine field. Tissue engineering approaches based on smart biomaterials, either natural or synthetic, are currently under experimental investigation as potential replacement solutions [49-51]. Moreover, the same tissue-engineered constructs can be applied as in vitro 3D models of inherited or acquired multifactorial diseases [52].

Such a variety of applications is reasonable since human pluripotent stem cells allow for the generation of an unlimited source of specialized cardiac cells, thus overcoming the previously described limitations with human primary cardiomyocyte cultures.

Even though, such possibilities result completely unprecedented before its adventure, iPSC technology needs further technical improvements in cell differentiation, above all for the cardiac lineage. The molecular route leading an iPSC to a cardiac myocyte is particularly long and needs to take into account the developmental steps observed in the embryo. For such a purpose, several differentiation protocols have been aimed to enrich a specific cell type in upscaling strategies [53]. Relatively incomplete cell maturation remains a considerable issue in the thrust to iPSC differentiation by conditioning with powerful cytokines. In most cardiac disease models reported in the literature, the use of sogenerated embryonic-like cardiac myocytes does not affect the ability to fully recapitulate the pathologic mechanisms in vitro and evaluate drug efficacy or side effects. Such immature state could possibly be detrimental for in vivo cell therapies or tissue engineering approaches, relying on iPSCs-differentiated cardiovascular progenitors, due to the potential generation of arrhythmic foci or teratoma.

\section{Modeling of Congenital Cardiac Diseases}

3.1. Brugada Syndrome. In 1992, Pedro and Joseph Brugada described a novel clinical manifestation characterized by right bundle branch block and persistent ST segment elevation, so severe to develop into fast polymorphic ventricular tachycardia, syncope, or unexpected cardiac death. This syndrome is associated with mutations in several genes, as SCN5A, GPD1-L, SCN1b, KCNE3, and SCN3B, respectively, encoding for the cardiac sodium channel, glycerol3-phosphate dehydrogenase 1-like, sodium channel beta-1 subunit, $I_{\mathrm{Ks}}$ and $I_{\text {to }}$ channels beta-subunit, and sodium channel beta-3 subunit. Among the forms described so far, the most of such autosomal dominant mutations induce a loss of protein functionality and/or reduced sodium current, while only a mutated $I_{\mathrm{Ks}}$ and $I_{\text {to }}$ channels beta-subunit has been correlated to a gain of function [54].

In order to reproduce experimentally the imbalanced currents of the phase 1 of the action potential observed in the heart of affected patients, many bioinformatic models have been suggested upon affected cardiomyocytes [55]. Since the real electrophysiological events in the disturbed action potential are still unknown, these cellular simulations tried to reproduce the increased transmural dispersion of repolarization across the ventricular wall, as formulated on the basis of former electrophysiological models and basic research evidences. The virtual epicardial, endocardial, and $\mathrm{M}$ cells mimicked the electrophysiological behavior of Brugada syndrome regarding the decreasing inward sodium current and the overwhelming outward $I_{\text {to }}$ ones, with a consequent decreased maximum AP peak value and delayed development of the characteristic notched morphology. Experimental confirmation of such proposed mechanism has been obtained in dog models either by ventricular wedge or by epicardium cooling [56-58]. The major interest of the right ventricle induced the formulation of a second hypothesis, reflecting the slowed impulse conduction consequent to the decreased sodium current [59]. Several mouse transgenic models have been created also for SCN5A, found mutated in almost $30 \%$ patients affected by Brugada syndrome. A substantial $I_{\mathrm{Na}}$ decrease and frequent mono- or biventricular arrhythmic events have been described in heterozygous 
mice [60]. Davis et al. were able to model the disease in vitro through multiple approaches relying on pluripotent stem cells of embryonic (mouse) and induced (mouse and human) origin. From an S5CNA-heterozygous mouse model $\left(\mathrm{Scn} 5 \mathrm{a}^{1798 \mathrm{insD} /+}\right)$, iPSCs, and ESCs (used to generate the same transgenic animal) were differentiated towards cardiac myocytes. While demonstrating an immature phenotype in respect to their counterparts harvested from the adult heterologous mice, such cells did show reduced and larger persistent sodium currents, responsible for slowed upstroke and prolonged AP, hallmarks of the syndrome. Cardiac myocytes, differentiated from reprogrammed dermal fibroblasts of a patient carrying a SCN5A mutation, equally demonstrated reduced sodium current density in respect to similar cells obtained from control hiPSCs [61].

\subsection{Catecholaminergic Polymorphic Ventricular Tachycar-} dia. In the electrocardiogram of patients affected by catecholaminergic polymorphic ventricular tachycardia (CPVT), a normal electrical activity is registered at rest, until physical or emotional stresses, as well as adrenergic stimulations trigger supraventricular arrhythmic events. Disease signs, as atrial fibrillation, ventricular extrasystole, and/or supraventricular tachycardia, can manifest during childhood or, for the first time, even in the adult life [62]. The pathophysiology of CPVT is correlated to abnormalities in the cardiomyocyte calcium handling, provoked by mutations in either ryanodine receptor 2 (RYR2), calsequestrin (CSQ2), or triadin (TRDN) genes. In conditions of calcium overload, each of the resulting mutated proteins induces the loss of the self-regulation ability by the sarcoplasmic reticulum, where they are located either in the lumen or across the membrane. As a result, the leakage of calcium during the diastole induces delayed afterdepolarizations (DADs) and triggered activity (TA) if such depolarizations are able to reach the threshold of sodium channel activation.

Structural studies upon the RYR2-encoded cardiac ryanodine receptor have demonstrated a $66 \%$ similarity in sequence to other RyR proteins, especially for its tetrameric composition. It passes across the sarcoplasmic reticulum membrane with the transmembrane domain of each subunit, encompassing also the channel pore, while the rest of the protein sequence composes the cytosolic domain [63]. The whole complex results in a 1:4 stoichiometric relation to FKBP12-binding proteins, thought to have a stabilizing role in the calcium channel [64].

Molecular screenings evidenced that the most frequent mutations interest a single nucleotide, bringing about single substitutions in three specific regions of the amino acid sequence, that is, the central and $\mathrm{N}$ - and C-terminal ones.

Stable inducible (noncardiac) HEK293 cells, planar lipid bilayers, and/or HL-1 cardiac myocytes have been used to simulate the behavior of several RyR2 mutations. The C-terminal Q4201R and I4867M, the central S2246L and R2474S, as well as the N-terminal R176Q (T2504M) and L433P demonstrated in all expression systems a higher propensity for spontaneous release of calcium in conditions of its store overload (SOIRC). As evidenced by studies on each single channel, such mutations were able to increase the luminal calcium activation $[65,66]$. On the contrary, few mutations, as the R4496C, demonstrated a higher sensitivity to cytosolic calcium activation [67].

In generated knock-in mouse models [68], the typical signs of CPVT, i.e., DADs and TAs, were reported. The real mechanism underlying the pathology is still unclear and at least two in silico models have been proposed: the unbinding from the accessory protein FKBP12.6 and the domain unzipping. In the first hypothesis, the RYR2 mutation generates a protein unable to correctly bind to FKBP12.6 and thus is more unstable. Some observations from R4496C knock-in mouse do not seem supportive on this regard [68]. While the first formalism is based on protein-protein interaction, domain unzipping relies on incorrect domaindomain binding. In particular, a mutated Ryr2, characterized by a weak interaction in between the $\mathrm{N}$-terminal and the central domains of the protein, would be more sensible to luminal or cytosolic calcium due to a destabilized closed state.

iPSCs were generated with the classical retroviral method from skin fibroblasts of a young female being, carrying a novel mutation, that is, S406L, in the RYR2 gene. CPVT iPSCs-differentiated cardiac myocytes displayed no differences in the ryanodine receptor localization in respect to control cells obtained from healthy iPSCs. Conversely, at the evaluation of AP, isoproterenol stimulation was able to trigger DADs and TAs. Calcium diastolic dispersion from the sarcoplasmic reticulum could be observed after catecholaminergic stimulation. Furthermore, calcium spark analyses revealed abnormal activity, characterized by increased frequency and longer duration. All of these observations confirm the ability of the CPVT iPSC model in recapitulating the pathologic phenotype and could be compatible in sustaining the domain unzipping model [69].

In the clinical practice, CPVT is pharmacologically treated with beta-blockers. More and more often, these are palliative treatments insufficient to control the instable activity of the heart, thus requiring the surgical implantation of cardioverter defibrillators. Recently, flecainide has demonstrated efficacy in preventing DADs in experimental models and its effects are currently under evaluation in a clinical trial [70, NCT01117454].

iPSC cardiac disease modeling has allowed to evaluate a novel potential drug therapy for CPVT, that is, dantrolene, a muscle relaxant clinically used for the treatment of malignant hyperthermia. Such hydantoin derivative was reported to act directly on mutated ryanodine receptor 1 , responsible for muscle spasticity in malignant hyperthermia patients after induction of general anesthesia [71]. When this drug was applied to isoproterenol-stimulated arrhythmic cardiac myocytes, derived by S406L CPVT iPSC differentiation, DADs and TAs were completely abolished [69].

3.3. Long QT Syndromes. Patients affected by LQT syndrome manifest an excessive prolongation in the repolarization phase, abnormal $\mathrm{T}$ waves, and early afterdepolarizations (EADs). The signs at the clinical presentation are syncope, ventricular arrhythmias, or sudden cardiac death, mostly linked to a preceding familiar history. 
Several forms of LQTs have been so far described, each characterized by mainly autosomal dominant mutations of potassium, sodium, or calcium channel and/or associated to other systemic dysfunctions [72].

Heterologous expression systems in HEK293 and Xenopus oocytes have been particularly advantageous to study the behavior of mutated human ether-à-go-go-related Gene, as in the case of hERG1 encoding the alpha subunit of the potassium channel $\mathrm{KCNH} 2$ and found mutated in LQT2. Such models allow the appraisal of drug efficacy for LQT treatment: the second-generation $(\mathrm{H}) 1$ receptor antagonist mizolastine is able to block HERG1 potassium channels [73].

Transgenic mice carrying an amino acid deletion in the SCN5A-encoded sodium channel can reproduce the same mutation described in LQT3 patients; however, clinical signs cannot be correlated since dissimilar [74].

Odening et al. described the transgenesis of rabbits to express the mutated KCNQ1 and $\mathrm{KCNH} 2$, responsible for LQT1 and LQT2, revealing the importance of the molecular substrate in the severity of the phenotypic effects observed [30].

LQT1, LQT2, LQT3, and Timothy syndrome, known also as LQT8 and characterized by a mutated calcium channelbased long QT, neurologic disorders, and syndactyly, were modeled with iPSCs derived from affected patients, verifying the distinctive APD prolongation and testing also several drug compounds already used in the clinical practice, as propranolol, or of novel generation, as PD-118057 [48, 61, 7578].

3.4. Arrhythmogenic Right Ventricular Cardiomyopathy. Arrhythmogenic right ventricular cardiomyopathy (ARVC) was electrocardiographically described for the first time in 1978 [79]. Unlike other arrhythmogenic inherited pathologies with intact heart tissue, such disease presents both dystrophic character and serious rhythm disturbances. A fibrofatty replacement of the myocardium is associated with the electrical instability of the right ventricle, even if an involvement of the left ventricle is not excluded. The prevalence of this congenital disease has been estimated to be $1: 2.000-$ 1:5.000. Affected patients may develop severe nonischemic ventricular arrhythmias evolving to sudden death [80]. In nearly half of ARVC cases, a genetic etiology is found. Mostly transmitted with autosomal dominant trait, inherited ARVC is induced by the mutation/s of desmosomal protein genes. Desmosome components, i.e., plakophilin-2, plakoglobin, desmoplakin, desmogleins, and other accessory or correlated proteins, as TMEM transmembrane protein, TGF $\beta 3$, and $\beta$ catenin, have found to be mutated in patients submitted to genetic screening $[81,82]$.

ARVC predictive models have been generated by implementing observational data with counterintuitive hypotheses, as the graphical representation formulated with the KEGGtranslator V2.3.0 software [83].

When the desmoplakin genetic variants, i.e., the $\mathrm{N}$ terminal encoding V30M, Q90R, and W233X, and the Cterminal encoding $\mathrm{R} 2834 \mathrm{H}$ found in ARVC patients, were introduced in the SCC-9 heterologous expression system (SCC-9 is a human tongue squamous carcinoma cell line), the resulting proteins of the first two genes showed defective ability to be expressed at the cell membrane, as confirmed by immunocytochemistry analyses. The same mutated proteins were prevented from binding to plakoglobin in coimmunoprecipitation analyses. Mice overexpressing V30M and Q90R died in early embryonic developmental phases [84].

Several transgenic mice have been generated either by targeted deletion or overexpression strategies and among other animal models, boxer dogs were also considered for ARVC modeling as natural carriers of the disease [29, 34]. In the former, the typical adipose tissue detected in humans was not evident. The latter developed a series of pathological events very close to the humans in terms of both fibrofatty tissue formation and electric instability.

The ARVC mechanism remains still unraveled: actually, the molecular alterations resulting in the replacement of a cardiomyocyte with an adipose cell are still unclear. Several theories have been suggested to explain this cell conversion. Lombardi et al. support the possibility of an adipose transdifferentiation of c-kit- and Scal-positive cardiac stem cells. They verified that cardiac progenitor cells of transgenic mice with a truncated plakoglobin could be easily committed to the adipocyte lineage in vitro. A canonical Wnt signaling impairment was proposed: mutated plakoglobin is not able to assemble at the desmosome and hence it translocates to the nucleus competing with $\beta$-catenin for the binding to TCF, necessary to activate targeted genes transcription. The adipose differentiation was experimentally blocked in cardiac progenitors by reactivating Wnt pathway with a specific GSK$3 \beta$ inhibitor, that is, $\mathrm{BIO}$. As confirmation of the adverse role of free plakoglobin, no change in the Wnt pathway could be demonstrated in conditions of null protein expression [85].

Recently, three human iPSC-based models of ARVC have been reported, all generated by patients carrying a plakophilin 2 mutation. Increased lipogenesis was always described in cardiac myocytes obtained by differentiated patient iPSCs in respect to their control counterpart. Moreover, abnormal plakoglobin nuclear translocation and/or upregulation of proadipogenic transcription factor peroxisome proliferatoractivated receptor gamma (PPAR- $\gamma$ ) and other adipogenesisrelated genes were demonstrated. The rescue of such phenotype could be realized by genetic engineering to overexpress the wild-type plakophilin 2 gene or with BIO cell conditioning [86-88].

\section{Final Remarks}

Most updated statistics of the American Heart Association reveal that every 39 seconds a person dies for cardiovascular disease (CVD), either acquired or congenital [1, 89]. With an expected increase of CVD prevalence in the next years, the need to identify and administer effective pharmacological or surgical therapies to affected patients is more and more urgent.

Even though the liability of theoretical formalisms, the interspecies differences in electrophysiological dynamics and/or the technical complexities in cell culture/differentiation, such in silico, in vitro, and in vivo modeling 
systems have been representing powerful instruments to achieve more information regarding the physiology of the whole cardiovascular apparatus in healthy and pathological settings.

This continuously evolving, integrative multiscale approach will render feasible in the future CVD therapeutics carefully tailored to the personal clinical requirements of the patient.

\section{Conflict of Interests}

The author declares that there is no conflict of interests regarding the publication of this paper.

\section{References}

[1] "Heart disease \& stroke statistics - 2013 update from the American Heart Association".

[2] F. M. Ashcroft, "From molecule to malady," Nature, vol. 440, no. 7083, pp. 440-447, 2006.

[3] M. Cerrone and S. G. Priori, "Genetics of sudden death: focus on inherited channelopathies," European Heart Journal, vol. 32, no. 17, pp. 2109-2120, 2011.

[4] A. L. Hodgkin and A. F. Huxley, "A quantitative description of membrane current and its application to conduction and excitation in nerve," The Journal of Physiology, vol. 117, no. 4, pp. 500-544, 1952.

[5] R. J. French and R. Horn, "Sodium channel gating: models, mimics, and modifiers," Annual Review of Biophysics and Bioengineering, vol. 12, pp. 319-356, 1983.

[6] F. H. Fenton and E. M. Cherry, "Models of cardiac cell," Scholarpedia, vol. 3, no. 8, article 1868, 2008.

[7] D. Noble and Y. Rudy, "Models of cardiac ventricular action potentials: iterative interaction between experiment and simulation," Philosophical Transactions of the Royal Society A: Mathematical, Physical and Engineering Sciences, vol. 359, no. 1783, pp. 1127-1142, 2001.

[8] R. E. McAllister, D. Noble, and R. W. Tsien, "Reconstruction of the electrical activity of cardiac Purkinje fibres," Journal of Physiology, vol. 251, no. 1, pp. 1-59, 1975.

[9] G. W. Beeler and H. Reuter, "Reconstruction of the action potential of ventricular myocardial fibres," Journal of Physiology, vol. 268, no. 1, pp. 177-210, 1977.

[10] D. DiFrancesco and C. Ojeda, "Properties of the current if in the sino-atrial node of the rabbit compared with those of the current iK, in Purkinje fibres," Journal of Physiology, vol. 308, pp. 353-367, 1980.

[11] D. DiFrancesco and D. Noble, "A model of cardiac electrical activity incorporating ionic pumps and concentration changes," Philosophical transactions of the Royal Society of London. Series B: Biological sciences, vol. 307, no. 1133, pp. 353-398, 1985.

[12] C.-H. Luo and Y. Rudy, "A model of the ventricular cardiac action potential. Depolarization, repolarization, and their interaction," Circulation Research, vol. 68, no. 6, pp. 1501-1526, 1991.

[13] C.-H. Luo and Y. Rudy, "A dynamic model of the cardiac ventricular action potential: I. Simulations of ionic currents and concentration changes," Circulation Research, vol. 74, no. 6, pp. 1071-1096, 1994.

[14] K. H. W. J. Ten Tusscher, D. Noble, P. J. Noble, and A. V. Panfilov, "A model for human ventricular tissue," American Journal of
Physiology: Heart and Circulatory Physiology, vol. 286, no. 4, pp. H1573-H1589, 2004.

[15] A. F. Huxley, "Muscle structure and theories of contraction," Progress in Biophysics and Biophysical Chemistry, vol. 7, pp. 255318, 1957.

[16] V. S. Markhasin, O. Solovyova, L. B. Katsnelson, Y. Protsenko, P. Kohl, and D. Noble, "Mechano-electric interactions in heterogeneous myocardium: development of fundamental experimental and theoretical models," Progress in Biophysics and Molecular Biology, vol. 82, no. 1-3, pp. 207-220, 2003.

[17] J. J. Rice, F. Wang, D. M. Bers, and P. P. de Tombe, "Approximate model of cooperative activation and crossbridge cycling in cardiac muscle using ordinary differential equations," Biophysical Journal, vol. 95, no. 5, pp. 2368-2390, 2008.

[18] S. A. Niederer, G. Plank, P. Chinchapatnam et al., "Lengthdependent tension in the failing heart and the efficacy of cardiac resynchronization therapy," Cardiovascular Research, vol. 89, no. 2, pp. 336-343, 2011.

[19] S. Kupershmidt, I. C.-H. Yang, M. Sutherland et al., "Cardiacenriched LIM domain protein fhl 2 is required to generate $\mathrm{I}_{\mathrm{Ks}}$ in a heterologous system," Cardiovascular Research, vol. 56, no. 1, pp. 93-103, 2002.

[20] S.-H. Jo, J. B. Youm, I. Kim, C. O. Lee, Y. E. Earm, and W.-K. Ho, "Blockade of HERG channels expressed in Xenopus oocytes by external $\mathrm{H}^{+}$," Pflügers Archiv, vol. 438, no. 1, pp. 23-29, 1999.

[21] P. Tammaro, K. Shimomura, and P. Proks, "Xenopus oocytes as a heterologous expression system for studying ion channels with the patch-clamp technique," Methods in Molecular Biology, vol. 491, pp. 127-139, 2008.

[22] W. J. Crumb and I. Cavero, "Patch-clamp studies of human cardiac ion channels in the evaluation of cardiac electrophysiological effects of compounds," Current Protocols in Pharmacology, vol. 10, unit 10.8, 2003.

[23] W. Crumb and I. Cavero, "QT interval prolongation by noncardiovascular drugs: issues and solutions for novel drug development," Pharmaceutical Science and Technology Today, vol. 2, no. 7, pp. 270-280, 1999.

[24] B. Jiang, G. Tang, K. Cao, L. Wu, and R. Wang, "Molecular mechanism for $\mathrm{H}_{2} \mathrm{~S}$-induced activation of $\mathrm{K}_{\mathrm{ATP}}$ channels," Antioxidants and Redox Signaling, vol. 12, no. 10, pp. 1167-1178, 2010.

[25] J. S. Mitcheson, J. C. Hancox, and A. J. Levi, "Action potentials, ion channel currents and transverse tubule density in adult rabbit ventricular myocytes maintained for 6 days in cell culture," Pflügers Archiv, vol. 431, no. 6, pp. 814-827, 1996.

[26] S. L. Jacobson, "Culture of spontaneously contracting myocardial cells from adult rats," Cell Structure and Function, vol. 2, no. 1, pp. 1-9, 1977.

[27] L. B. Bugaisky and R. Zak, "Differentiation of adult rat cardiac myocytes in cell culture," Circulation Research, vol. 64, no. 3, pp. 493-500, 1989.

[28] P. R. Fox, B. J. Maron, C. Basso, S.-K. Liu, and G. Thiene, "Spontaneously occurring arrhythmogenic right ventricular cardiomyopathy in the domestic cat: a new animal model similar to the human disease," Circulation, vol. 102, no. 15, pp. 1863-1870, 2000.

[29] C. Basso, P. R. Fox, K. M. Meurs et al., "Arrhythmogenic right ventricular cardiomyopathy causing sudden cardiac death in boxer dogs: a new animal model of human disease," Circulation, vol. 109, no. 9, pp. 1180-1185, 2004. 
[30] K. E. Odening, M. Kirk, M. Brunner et al., "Electrophysiological studies of transgenic long QT type 1 and type 2 rabbits reveal genotype-specific differences in ventricular refractoriness and His conduction," American Journal of Physiology: Heart and Circulatory Physiology, vol. 299, no. 3, pp. H643-H655, 2010.

[31] F. Charpentier, A. Bourgé, and J. Mérot, "Mouse models of SCN5A-related cardiac arrhythmias," Progress in Biophysics and Molecular Biology, vol. 98, no. 2-3, pp. 230-237, 2008.

[32] B. London, L. C. Baker, P. Petkova-Kirova, J. M. Nerbonne, B.-R. Choi, and G. Salama, "Dispersion of repolarization and refractoriness are determinants of arrhythmia phenotype in transgenic mice with long QT,' Journal of Physiology, vol. 578, no. 1, pp. 115-129, 2007.

[33] D. E. Gutstein, G. E. Morley, H. Tamaddon et al., "Conduction slowing and sudden arrhythmic death in mice with cardiacrestricted inactivation of connexin43," Circulation Research, vol. 88, no. 3, pp. 333-339, 2001.

[34] E. M. Lodder and S. Rizzo, "Mouse models in arrhythmogenic right ventricular cardiomyopathy," Frontiers in Electrophysiology, vol. 3, article 221, 2012.

[35] T. O’Hara and Y. Rudy, “Quantitative comparison of cardiac ventricular myocyte electrophysiology and response to drugs in human and nonhuman species," American Journal of Physiology: Heart and Circulatory Physiology, vol. 302, no. 5, pp. H1023H1030, 2012.

[36] I. M. Ayoub, J. Kolarova, Z. Yi et al., "Sodium-hydrogen exchange inhibition during ventricular fibrillation: beneficial effects on ischemic contracture, action potential duration, reperfusion arrhythmias, myocardial function, and resuscitability," Circulation, vol. 107, no. 13, pp. 1804-1809, 2003.

[37] G.-R. Li, X.-L. Du, Y. L. Siow, O. Karmin, H.-F. Tse, and C.P. Lau, "Calcium-activated transient outward chloride current and phase 1 repolarization of swine ventricular action potential," Cardiovascular Research, vol. 58, no. 1, pp. 89-98, 2003.

[38] J. M. Nerbonne, "Studying cardiac arrhythmias in the mouse-a reasonable model for probing mechanisms?" Trends in Cardiovascular Medicine, vol. 14, no. 3, pp. 83-93, 2004.

[39] A. M. From, J. J. Maleszewski, and C. S. Rihal, "Current status of endomyocardial biopsy," Mayo Clinic Proceedings, vol. 86, no. 11, pp. 1095-1102, 2011.

[40] R. K. Li, L. C. Tumiati, R. D. Weisel, and D. A. G. Mickle, "Isolation of cardiomyocytes from human myocardium for primary cell culturing," Journal of Tissue Culture Methods, vol. 15, no. 3, pp. 147-154, 1993.

[41] J. A. Thomson, "Embryonic stem cell lines derived from human blastocysts," Science, vol. 282, no. 5391, pp. 1145-1147, 1998.

[42] K. Takahashi and S. Yamanaka, "Induction of pluripotent stem cells from mouse embryonic and adult fibroblast cultures by defined factors," Cell, vol. 126, no. 4, pp. 663-676, 2006.

[43] K. Takahashi, K. Tanabe, M. Ohnuki et al., "Induction of pluripotent stem cells from adult human fibroblasts by defined factors," Cell, vol. 131, no. 5, pp. 861-872, 2007.

[44] T. Seki, S. Yuasa, and K. Fukuda, "Derivation of induced pluripotent stem cells from human peripheral circulating $\mathrm{T}$ cells," Current Protocols in Stem Cell Biology, vol. 18, pp. 4A.3.14A.3.9, 2011.

[45] W. Yang, J. A. Mills, S. Sullivan, Y. Liu, D. L. French, and P. Gadue, "iPSC reprogramming from human peripheral blood using sendai virus mediated gene transfer," in StemBook, Harvard Stem Cell Institute, Cambridge, Mass, USA, 2008-2012.
[46] C. Mauritz, K. Schwanke, M. Reppel et al., "Generation of functional murine cardiac myocytes from induced pluripotent stem cells," Circulation, vol. 118, no. 5, pp. 507-517, 2008.

[47] A. Moretti, M. Bellin, C. B. Jung et al., "Mouse and human induced pluripotent stem cells as a source for multipotent $\mathrm{Isl}^{+}$ cardiovascular progenitors," FASEB Journal, vol. 24, no. 3, pp. 700-711, 2010.

[48] C. Terrenoire, K. Wang, K. W. Tung et al., "Induced pluripotent stem cells used to reveal drug actions in a long QT syndrome family with complex genetics," The Journal of General Physiology, vol. 141, no. 1, pp. 61-72, 2013.

[49] A. Margariti, B. Winkler, E. Karamariti et al., "Direct reprogramming of fibroblasts into endothelial cells capable of angiogenesis and reendothelialization in tissue-engineered vessels," Proceedings of the National Academy of Sciences of the United States of America, vol. 109, no. 34, pp. 13793-13798, 2012.

[50] W.-H. Zimmermann and T. Eschenhagen, "Embryonic stem cells for cardiac muscle engineering," Trends in Cardiovascular Medicine, vol. 17, no. 4, pp. 134-140, 2007.

[51] S. L. J. Ng, K. Narayanan, S. Gao, and A. C. A. Wan, "Lineage restricted progenitors for the repopulation of decellularized heart," Biomaterials, vol. 32, no. 30, pp. 7571-7580, 2011.

[52] C. Rao, H. Barratt, T. Prodromakis, and C. M. Terracciano, "Tissue engineering techniques in cardiac repair and disease modelling," Current Pharmaceutical Design, 2013.

[53] C. L. Mummery, J. Zhang, E. S. Ng, D. A. Elliott, A. G. Elefanty, and T. J. Kamp, "Differentiation of human embryonic stem cells and induced pluripotent stem cells to cardiomyocytes: a methods overview," Circulation Research, vol. 111, no. 3, pp. 344358, 2012.

[54] A. L. George Jr., "Molecular and genetic basis of sudden cardiac death," The Journal of Clinical Investigation, vol. 123, no. 1, pp. 75-83, 2013.

[55] L. Xia, Y. Zhang, and X. H. Lu, "Cellular modeling and simulation of brugada syndrome," in Proceedings of the Computers in Cardiology (CinC '05), pp. 1001-1004, Lyon, France, September 2005.

[56] G.-X. Yan and C. Antzelevitch, "Cellular basis for the Brugada syndrome and other mechanisms of arrhythmogenesis associated with ST-segment elevation," Circulation, vol. 100, no. 15, pp. 1660-1666, 1999.

[57] J. M. Fish and C. Antzelevitch, "Cellular and ionic basis for the sex-related difference in the manifestation of the Brugada syndrome and progressive conduction disease phenotypes," Journal of Electrocardiology, vol. 36, supplement 1, pp. 173-179, 2003.

[58] K. Nishida, A. Fujiki, K. Mizumaki et al., "Canine model of Brugada syndrome using regional epicardial cooling of the right ventricular outflow tract," Journal of Cardiovascular Electrophysiology, vol. 15, no. 8, pp. 936-941, 2004.

[59] P. G. Meregalli, A. A. M. Wilde, and H. L. Tan, "Pathophysiological mechanisms of Brugada syndrome: depolarization disorder, repolarization disorder, or more?" Cardiovascular Research, vol. 67, no. 3, pp. 367-378, 2005.

[60] G. A. Papadatos, P. M. R. Wallerstein, C. E. G. Head et al., "Slowed conduction and ventricular tachycardia after targeted disruption of the cardiac sodium channel gene Scn5a," Proceedings of the National Academy of Sciences of the United States of America, vol. 99, no. 9, pp. 6210-6215, 2002.

[61] R. P. Davis, S. Casini, C. W. van den Berg et al., "Cardiomyocytes derived from pluripotent stem cells recapitulate electrophysiological characteristics of an overlap syndrome of cardiac sodium 
channel disease," Circulation, vol. 125, no. 25, pp. 3079-3091, 2012.

[62] M. Cerrone, C. Napolitano, and S. G. Priori, "Catecholaminergic polymorphic ventricular tachycardia: a paradigm to understand mechanisms of arrhythmias associated to impaired $\mathrm{Ca}^{2+}$ regulation," Heart Rhythm, vol. 6, no. 11, pp. 1652-1659, 2009.

[63] F. J. Amador, P. B. Stathopulos, M. Enomoto, and M. Ikura, "Ryanodine receptor calcium release channels: lessons from structure-function studies," FEBS Journal, vol. 280, no. 21, pp. 5456-5470, 2013.

[64] T. Guo, R. L. Cornea, S. Huke et al., "Kinetics of FKBP12.6 binding to ryanodine receptors in permeabilized cardiac myocytes and effects on Ca sparks," Circulation Research, vol. 106, no. 11, pp. 1743-1752, 2010.

[65] D. Jiang, R. Wang, B. Xiao et al., "Enhanced store overloadinduced $\mathrm{Ca}^{2+}$ release and channel sensitivity to luminal $\mathrm{Ca}^{2+}$ activation are common defects of RyR2 mutations linked to ventricular tachycardia and sudden death," Circulation Research, vol. 97, no. 11, pp. 1173-1181, 2005.

[66] D. Jiang, P. P. Jones, D. R. Davis et al., "Characterization of a novel mutation in the cardiac ryanodine receptor that results in catecholaminergic polymorphic ventricular tachycardia," Channels, vol. 4, no. 4, pp. 302-310, 2010.

[67] S. G. Priori, C. Napolitano, N. Tiso et al., "Mutataions in the cardiac ryanodine receptor gene (hRyR2) underlie catecholaminergic polymorphic ventricular tachycardia," Circulation, vol. 103, no. 2, pp. 196-200, 2001.

[68] N. Liu, B. Colombi, M. Memmi et al., "Arrhythmogenesis in catecholaminergic polymorphic ventricular tachycardia: insights from a RyR2 R4496C knock-in mouse model," Circulation Research, vol. 99, no. 3, pp. 292-298, 2006.

[69] C. B. Jung, A. Moretti, M. Mederos y Schnitzler et al., "Dantrolene rescues arrhythmogenic RYR2 defect in a patient-specific stem cell model of catecholaminergic polymorphic ventricular tachycardia," EMBO Molecular Medicine, vol. 4, no. 3, pp. 180191, 2012.

[70] H. Watanabe, C. van der Werf, F. Roses-Noguer et al., "Effects of flecainide on exercise-induced ventricular arrhythmias and recurrences in genotype-negative patients with catecholaminergic polymorphic ventricular tachycardia," Heart Rhythm, vol. 10, no. 4, pp. 542-547, 2013.

[71] C. M. Friesen, J. B. Brodsky, and M. F. Dillingham, "Successful use of dantrolene sodium in human malignant hyperthermia syndrome: a case report," Canadian Anaesthetists Society Journal, vol. 26, no. 4, pp. 319-321, 1979.

[72] L. Crotti, G. Celano, F. Dagradi, and P. J. Schwartz, "Congenital long QT syndrome," Orphanet Journal of Rare Diseases, vol. 3, no. 1 , article 18, 2008.

[73] M. Taglialatela, A. Pannaccione, P. Castaldo, G. Giorgio, and L. Annunziato, "Inhibition of HERG1 $\mathrm{K}^{+}$channels by the novel second-generation antihistamine mizolastine," British Journal of Pharmacology, vol. 131, no. 6, pp. 1081-1088, 2000.

[74] D. Nuyens, M. Stengl, S. Dugarmaa et al., "Abrupt rate accelerations or premature beats cause life-threatening arrhythmias in mice with long-QT3 syndrome," Nature Medicine, vol. 7, no. 9, pp. 1021-1027, 2001.

[75] A. Moretti, M. Bellin, A. Welling et al., "Patient-specific induced pluripotent stem-cell models for long-QT syndrome," The New England Journal of Medicine, vol. 363, no. 15, pp. 1397-1409, 2010.

[76] I. Itzhaki, L. Maizels, I. Huber et al., "Modelling the long QT syndrome with induced pluripotent stem cells," Nature, vol. 471, no. 7337, pp. 225-230, 2011.
[77] E. Matsa, D. Rajamohan, E. Dick et al., "Drug evaluation in cardiomyocytes derived from human induced pluripotent stem cells carrying a long QT syndrome type 2 mutation," European Heart Journal, vol. 32, no. 8, pp. 952-962, 2011.

[78] S. P. Paşca, T. Portmann, I. Voineagu et al., "Using iPSCderived neurons to uncover cellular phenotypes associated with Timothy syndrome," Nature Medicine, vol. 17, no. 12, pp. 16571662, 2011.

[79] R. Frank, G. Fontaine, J. Vedel et al., "Electrocardiologie de quatre cas de dysplasie ventriculaire droite arythmogene," Archives des Maladies du Coeur et des Vaisseaux, vol. 71, pp. 963-972, 1978.

[80] G. Thiene, D. Corrado, and C. Basso, "Arrhythmogenic right ventricular cardiomyopathy/dysplasia," Orphanet Journal of Rare Diseases, vol. 2, no. 1, article 45, 2007.

[81] M. A. Schwarz, K. Owaribe, J. Kartenbeck, and W. W. Franke, "Desmosomes and hemidesmosomes: constitutive molecular components," Annual Review of Cell Biology, vol. 6, pp. 461-491, 1990.

[82] A. Rampazzo, "Genetic bases of arrhythmogenic right ventricular cardiomyopathy," Heart International, vol. 2, pp. 17-26, 2006.

[83] C. Wrzodek, A. Dräger, and A. Zell, "Graphical representation of "Arrhythmogenic Right Ventricular Cardiomyopathy (ARVC) (Ornithorhynchus anatinus (platypus))," Bioinformatics, vol. 27, pp. 2314-2315, 2011.

[84] Z. Yang, N. E. Bowles, S. E. Scherer et al., "Desmosomal dysfunction due to mutations in desmoplakin causes arrhythmogenic right ventricular dysplasia/cardiomyopathy," Circulation Research, vol. 99, no. 6, pp. 646-655, 2006.

[85] R. Lombardi, M. da Graca Cabreira-Hansen, A. Bell, R. R. Fromm, J. T. Willerson, and A. J. Marian, "Nuclear plakoglobin is essential for differentiation of cardiac progenitor cells to adipocytes in arrhythmogenic right ventricular cardiomyopathy," Circulation Research, vol. 109, no. 12, pp. 1342-1353, 2011.

[86] D. Ma, H. Wei, J. Lu et al., "Generation of patient-specific induced pluripotent stem cell-derived cardiomyocytes as a cellular model of arrhythmogenic right ventricular cardiomyopathy," European Heart Journal, vol. 34, no. 15, pp. 1122-1133, 2013.

[87] C. Kim, J. Wong, J. Wen et al., "Studying arrhythmogenic right ventricular dysplasia with patient-specific iPSCs," Nature, vol. 494, no. 7435, pp. 105-110, 2013.

[88] O. Caspi, I. Huber, A. Gepstein et al., "Modeling of arrhythmogenic right ventricular cardiomyopathy with human induced pluripotent stem cells," Circulation: Cardiovascular Genetics, vol. 6, no. 6, pp. 557-568, 2013.

[89] V. L. Roger, A. S. Go, D. M. Lloyd-Jones et al., "Executive summary: heart disease and stroke statistics-2012 update: a report from the American Heart Association," Circulation, vol. 125, no. 1, pp. 188-197, 2012. 


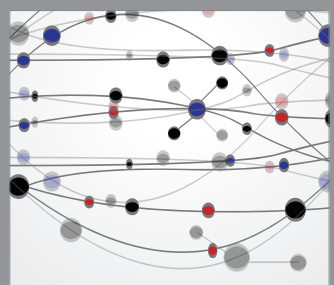

The Scientific World Journal
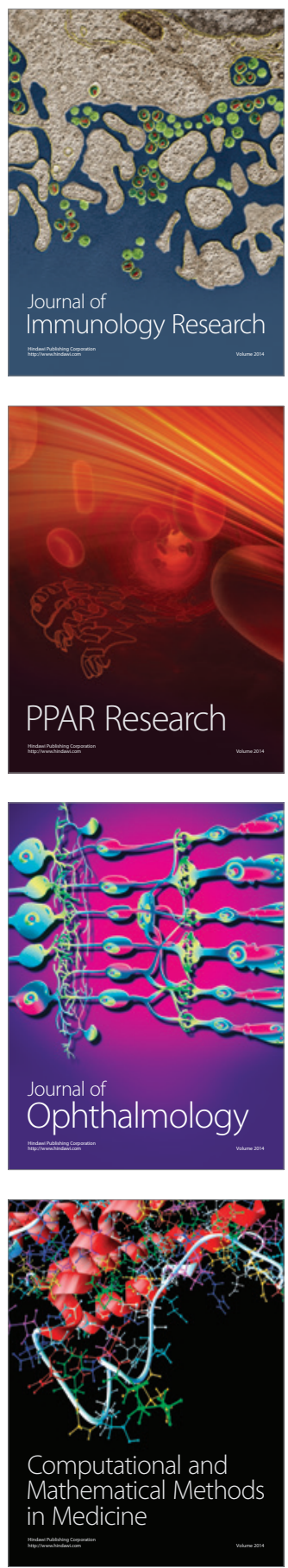

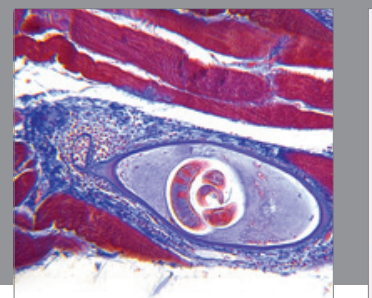

Gastroenterology

Research and Practice
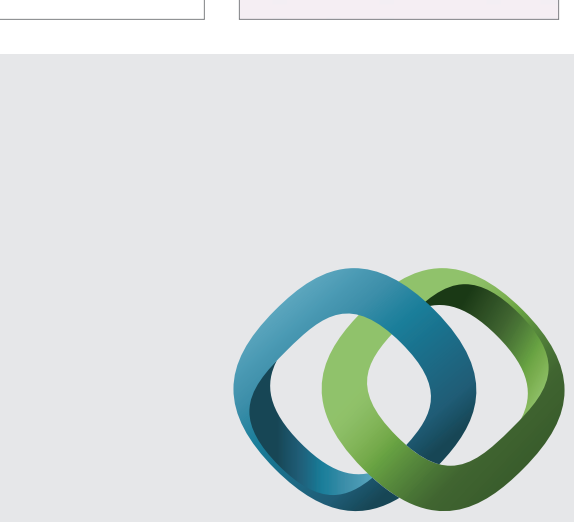

\section{Hindawi}

Submit your manuscripts at

http://www.hindawi.com
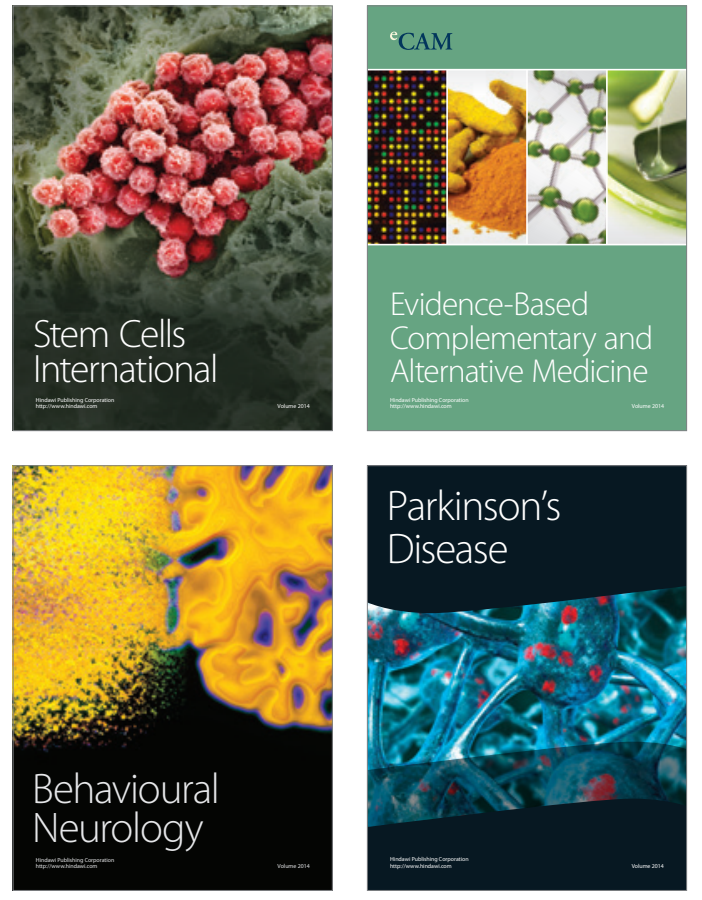
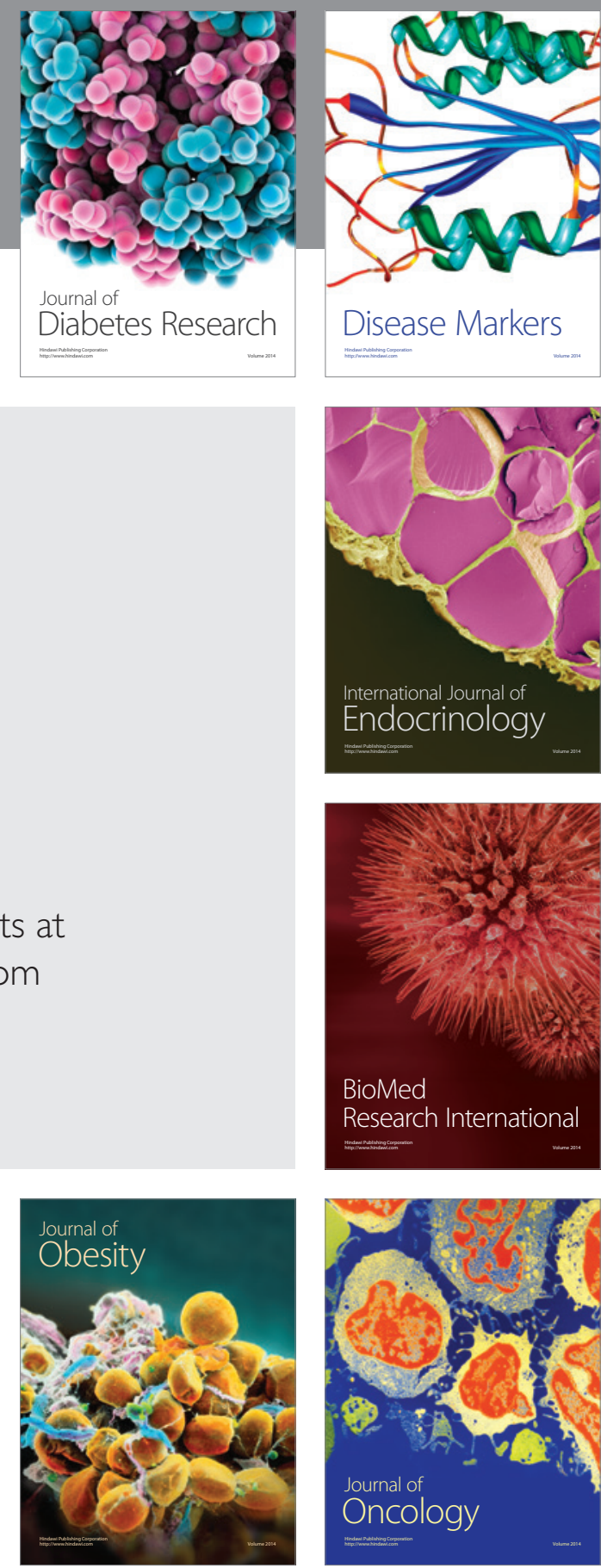

Disease Markers
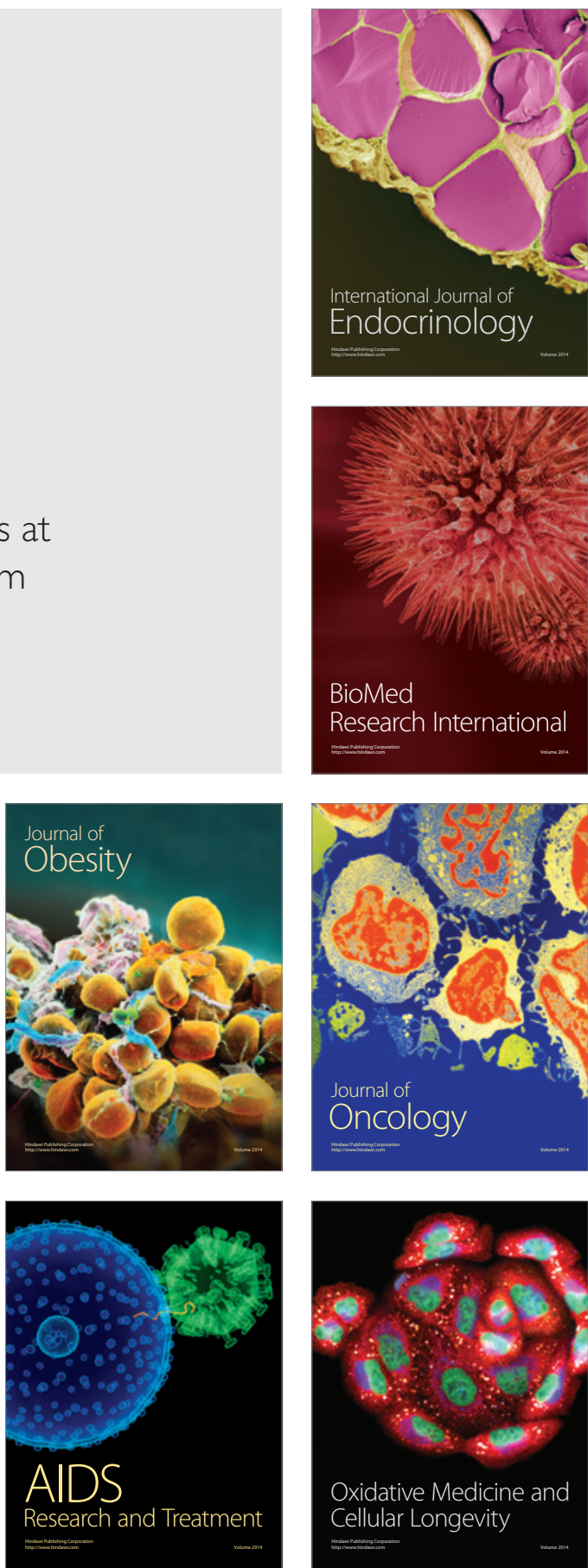